\title{
The block-like behavior of Anatolia envisaged in the modeled and geodetic strain rates
}

\author{
Ivone Jiménez-Munt and Roberto Sabadini \\ Geofisica, Department of Earth Sciences, University of Milan, Italy
}

Received 27 July 2002; revised 9 September 2002; accepted 10 September 2002; published 25 October 2002.

[1] The geodetic velocity and strain rate patterns in Anatolia constrain the rheology of the lithosphere, once compared with thin shell finite element tectonic model predictions. Geodetic and modeled deformation favors a hard lithospheric rheology, responsible for the high EastWest horizontal velocities in the center of Anatolia, between $39^{\circ}-41^{\circ} \mathrm{N}$, in proximity of the North Anatolian Fault. The block like behavior of Anatolia, due to the hard lithospheric rheology, is responsible for the low geodetic and modeled strain rates in the center of the peninsula. The low strain rate is well correlated with the region of low release of seismic energy between $33^{\circ}-36^{\circ} \mathrm{E}$ and $38^{\circ}-$ $40^{\circ} \mathrm{N}$. The high seismic and geodetic strain rate region in the far west part of the NAF is also well reproduced by the modeling. INDEX TERMS: 8107 Tectonophysics: Continental neotectonics; 8120 Tectonophysics: Dynamics of lithosphere and mantle-general; 8159 Tectonophysics: Evolution of the Earth: Rheology - crust and lithosphere; 8168 Tectonophysics: Evolution of the Earth: Stresses - general. Citation: Jiménez-Munt, I., and R. Sabadini, The block-like behavior of Anatolia envisaged in the modeled and geodetic strain rates, Geophys. Res. Lett., 29(20), 1978, doi:10.1029/2002GL015995, 2002.

\section{Introduction}

[2] Convergence between African, Arabian and European plates leads to a complex tectonics, characterized by the fingerprints of continental collision and subduction along the plate boundaries and surrounding regions. The motion of Anatolia is bounded in the north by the right-lateral North Anatolia Fault (NAF) and in the southeast by the left-lateral East Anatolia Fault (EAF) (Figure 1). A large uncertainty remains on the active tectonics in this zone, although the kinematics of Anatolia is generally explained by emphasizing either its westward motion [Dewey and Sengor, 1979; Taymaz et al., 1991] or the occurrence of roll-back in the southern part of the Hellenic subduction [Le Pichon and Angelier, 1979; Wortel and Spakman, 2000]. As a first approximation the relative motions are described through rigid plates [McKenzie, 1970; Le Pichon et al., 1995]. However, a rigid plate model is not capable to explain the observed intraplate deformations [Reilinger et al., 1997]. Some simulations of the velocity field on this area have been done using thin plate elastic [p.e: Meijer and Wortel, 1996; Lundgren et al., 1998] or thin plate viscous models [Cianetti et al., 2001].

[3] Detailed knowledge of the kinematics of plate motions and deformations is necessary but not sufficient to uniquely constrain the active tectonics of Anatolia. The comprehension of the dynamics of contemporary deformation in this complex area requires in fact the knowledge of the rheology of the plates. A finite element model of the whole Mediterranean by Jiménez-Munt et al. [2002], from Gibraltar to Anatolia, has shown that the zeroth order deformation pattern, as envisaged in the geodetic strain and in the release of seismic energy, can be explained in terms of continental collision and subduction in the Calabrian and Hellenic Arcs. The present analysis, based on the finite element approach used by Jiménez-Munt et al. [2002], develops new issues related to the effects of rheology of the lithosphere. Particular attention is drawn on the effects of the stiffening of the Anatolia block, on the modeled velocity field and strain rate, to see the extent to which GPS data and seismic strain allow to constrain the mechanical properties of Anatolia.

\section{Seismic Strain Rate}

[4] Figure 1 shows the seismic strain rate in Anatolia, based on the procedure described by Jiménez-Munt et al. [2001] computed for the entire Mediterranean [Jiménez-Munt et al., 2002]. The seismic strain rate has been calculated using the methodology described by Kostrov [1974], who proposed that the strain rate in a given area is proportional to the sum of earthquake scalar seismic moments. The seismic moment has been calculated from the surface magnitude, based on the National Earthquake Information Center (NEIC) catalogue. The seismic strain rate is subject to large variations from the western to the eastern part of Anatolia. The maximum values are located in the Aegean and in the western and eastern parts of Anatolia, at the intersection of the EAF and SAF, with a seismic strain rate minimum in the center, indicative of low intraplate deformation.

\section{Numerical Modeling}

[5] The velocity field is obtained from the SHELLS thin-shell finite element program developed by Bird [1999], within the frame of the general model described in Jiménez-Munt et al. [2002] for the whole Mediterranean. The modeling provides the steady state velocity and strain rates, corresponding to the geometries of the plates and to their relative motions, as given by NUVEL-1A [DeMets et al., 1994]. The local value of the lithospheric strength is obtained by the integration in the vertical direction of the stress envelope, within the assumption that the rheological parameters are constant throughout the model; laterals strength variations are thus due to crustal and lithospheric thickness heterogeneities. The effects of subduction on the geodetic and seismic strain are modeled by means of veloc- 


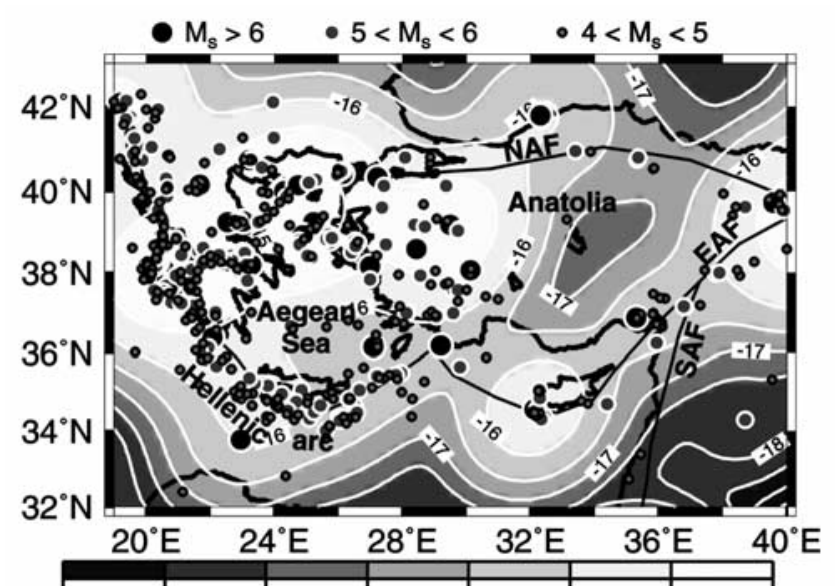

$\begin{array}{llllllll}-21.0 & -18.5 & -17.5 & -17.0 & -16.5 & -16.0 & -15.5 & -14.0\end{array}$ $\log \left(\right.$ seismic strain rate $\left.\left(\mathrm{s}^{-1}\right)\right)$

Figure 1. Seismicity with magnitude $\mathrm{M}_{\mathrm{s}}$ from NEIC Catalogue (1903-1999) and seismic strain rate [JiménezMunt et al., 2002].

ities, applied in the trench regions at the subducting and overthrusting plates, as shown in Figure 2a and by JiménezMunt et al. [2002]. The rectangle in Figure 2a indicates the area under study in the present analysis embedded within the global finite element model of the whole Mediterranean. This study focuses on the effects of the rheological stratification of the lithosphere. The strength of the lithosphere, is varied modifying the rheological parameters in the whole model, in order to enlighten the effects of creep or fragile behavior. In the central part of Anatolia, characterized by a crustal thickness of $28 \mathrm{~km}$ as inferred from topography and heat flow data [Jiménez-Munt et al., 2002], this strength variation results into a change from $0.87 \times 10^{12}$ to $9.3 \times 10^{12} \mathrm{~N} / \mathrm{m}$. The various lithospheric strengths have been obtained using different sets of rheological parameters based on the studies summarized by Kirby [1983] and on the compilation by Fernàndez and Ranalli [1997].

[6] The faults represented in Figure 1 are simulated by double nodes, with a low friction coefficient of 0.05 , which provides the best correlation between the geodetic, seismic and stress data at the global scale of the Mediterranean [Jiménez-Munt et al., 2002].

\subsection{Velocity Pattern}

[7] The GPS velocities from McClusky et al. [2000] and the modeled ones with respect Eurasia, are shown in Figures $2 \mathrm{~b}$ and $2 \mathrm{c}$, for two different lithospheric strengths, corresponding in the center of Anatolia to 3.6 and $9.3 \times 10^{12} \mathrm{~N} / \mathrm{m}$.

[8] This figure provides an image of the counterclockwise rotation of the velocity field from the eastern Anatolian fault to the Aegean sea, both in the geodetic data and in modeled results, with convergence almost completely absorbed in the NAF. The counterclockwise rotation of the velocity field is due to three major causes: the NNW push from the Arabian plate, the strong lithosphere discontinuity represented by the NAF and the suction forces exerted by the Hellenic arc subduction. In Figure 2b, the modeled velocity is slowly decreasing from the center of the Anatolian peninsula to the north, while the geodetic data show high velocities from the center of the peninsula to the
NAF: the medium rheology fails to reproduce the high EW directed velocities from the center of Anatolia to $40^{\circ} \mathrm{N}$. Although a relatively low friction coefficient characterizes the NAF, the tapering to zero of the modeled velocity in proximity of the NAF is due to the low effective viscosity of the soft lithosphere. On contrary, the hard lithospheric rheology (Figure 2c) carries into coincidence the high geodetic and modeled velocities, as shown by the close agreement between the black and grey arrows between $39^{\circ}$ and $41^{\circ} \mathrm{N}$. In the eastern part of Anatolia, the hard rheology does not improve the azimuthal misfit between observed and modeled velocities, the last ones being more north oriented than the observed ones, due to the proximity of this region to the boundary where the push from Arabia is applied.

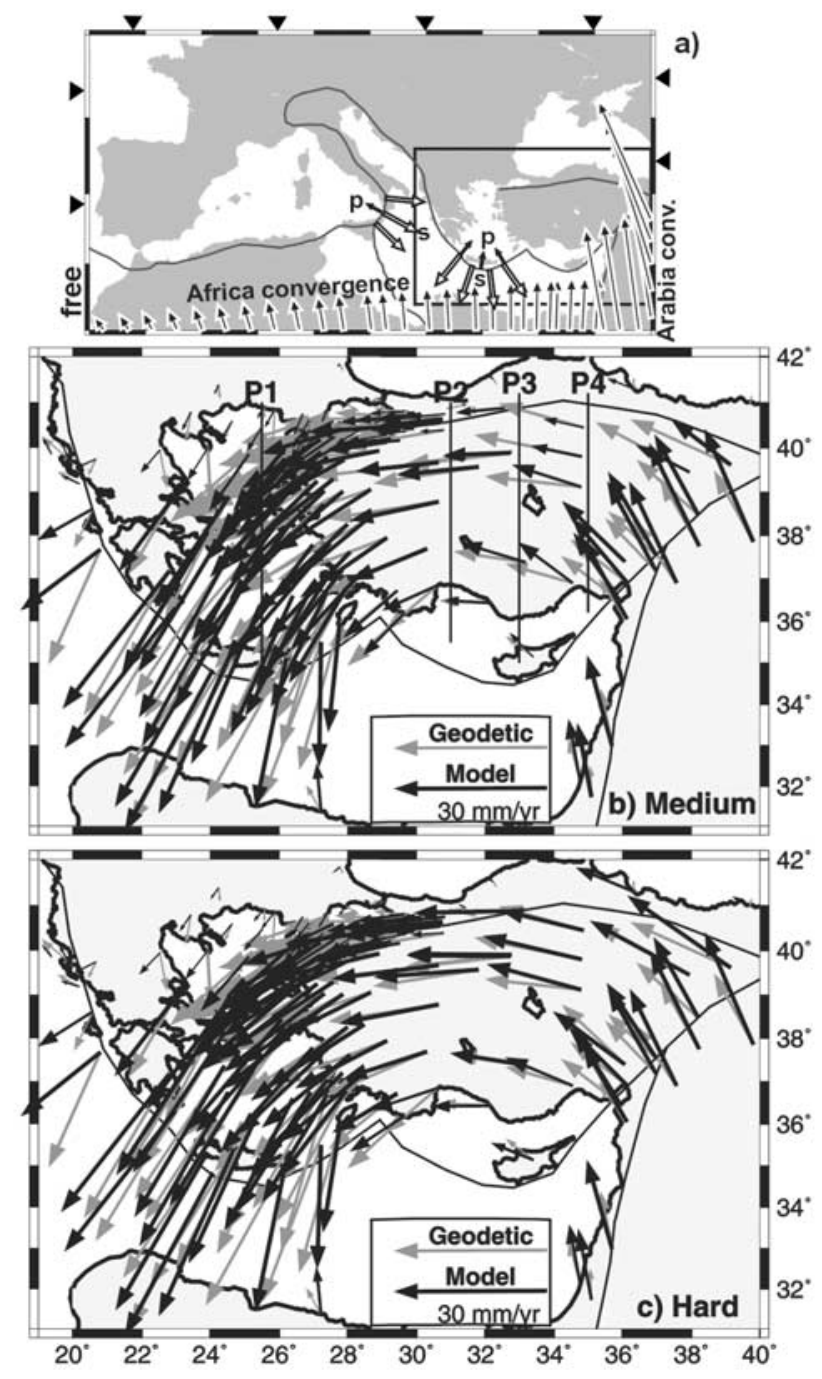

Figure 2. (a) Global model considered [Jiménez-Munt et al., 2002] with the boundary conditions: Africa and Arabia convergence from Nuvel-1A and slab pull $(\mathrm{p})$ and suction (s) forces in the Calabrian and Aegean Arcs. (b) Modeled (black arrows) and geodetic GPS velocities from McClusky et al. [2000] (grey arrows) for a typical lithospheric rheology. The lines on panel one (P1, P2, P3 and P4) correspond to the velocity profiles shown in Figure 3. (c) for a hard lithospheric rheology. 

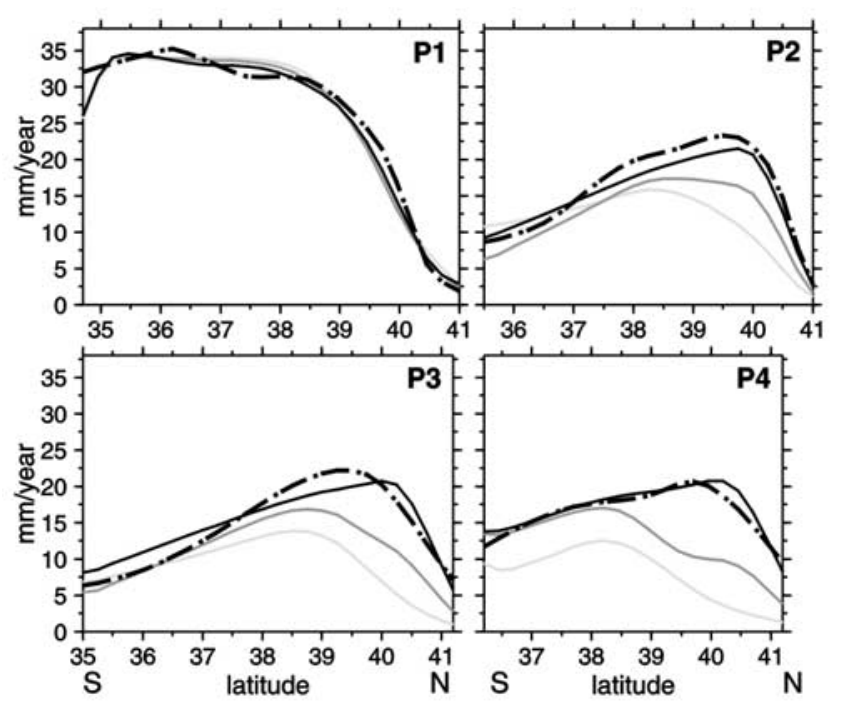

Figure 3. S-N profiles of the velocity modulus for three different lithospheric strengths. Discontinuous lines correspond to the measured geodetic velocity. The black line represents the strongest lithosphere, the intermediate grey the medium strength lithosphere and the light grey line the weakest lithosphere. The strengths of the lithosphere in the center Anatolia (with $28 \mathrm{~km}$ of crust and a heat flow of 70 $\mathrm{mW} / \mathrm{m}^{2}$ ) are $0.87,3.61,9.3 \times 10^{12} \mathrm{~N} / \mathrm{m}$.

[9] Figure 3 shows the modulus of the velocity along the four S-N profiles (P1, P2, P3 and P4 represented in Figure 2b) for the geodetic data and three numerical models, for soft, medium and hard lithospheric strengths. The geodetic velocity profiles have been obtained by interpolation of the geodetic data, made possible by the large number of observations in the area and by the absence of important structural discontinuities. The velocity pattern along the western profile P1 crossing the Aegean sea, carrying the highest velocity of about $30 \mathrm{~mm} / \mathrm{yr}$ (top, left panel) differs substantially from those crossing the Anatolia Peninsula (P2, P3 and P4). Along $\mathrm{P} 1$, high values are obtained between $35^{\circ}$ and $38.5^{\circ} \mathrm{N}$ latitude, due to the combination of the Arabia push and the SW directed suction force active in the Hellenic arc. A decrease from $30 \mathrm{~mm} / \mathrm{yr}$ to about $2 \mathrm{~mm} / \mathrm{yr}$ is obtained between $38.5^{\circ} \mathrm{N}$ and $40.5^{\circ} \mathrm{N}$. The numerical models reproduce this decrease well, indicating that velocity predictions do not show any sensitivity to rheology variations, and that the major controlling factor of the deformation is the mechanism of trench suction in the Hellenic arc.

[10] The behavior of the velocity pattern in the center of Anatolia is completely different (Figure 3, P2, P3, P4). In fact, the geodetic velocities are essentially E-W oriented, and the maximum value of $23 \mathrm{~mm} /$ year, is located between $39^{\circ} \mathrm{N}$ and $40^{\circ} \mathrm{N}$. The geodetic velocity increases smoothly from south to north until the maximum value is reached, after which it drops quickly to the level of $10 \mathrm{~mm} / \mathrm{yr}$ at most. The modeled velocity portrays a quite different behavior along these profiles, depending on the lithospheric strength. Only the hard lithospheric rheology reproduces well the geodetic data, as shown by the overlapping of the black (modeled) and dashed (geodetic) curves in the explored range of latitudes. The soft lithospheric rheology fails to reproduce the velocity pattern along the profiles within Anatolia, in terms of the velocity maximum and of its location along latitude. In fact, these models predict a too low value of the velocity maximum of $17 \mathrm{~mm} / \mathrm{yr}$ at the most, located between $38^{\circ} \mathrm{N}-39^{\circ} \mathrm{N}$, rather than $23 \mathrm{~mm} / \mathrm{yr}$ at $39^{\circ} \mathrm{N}-40^{\circ} \mathrm{N}$ along the three profiles.

\subsection{Horizontal Strain Rate Pattern}

[11] Figure 4 compares, for the hard rheology, the geodetic and the modeled horizontal strain rate tensor in the Aegean sea and in the Anatolia Peninsula. The method to calculate the eigenvectors and eigenvalues of the strain rate is described in detail by Devoti et al. [2001] and JiménezMunt et al. [2002], where the strain rate is calculated from the horizontal velocity components at the vertices of the triangles. These quantities represent first order estimates of the strain rate within the area embedded within the triangles with vertices at sites where the geodetic data are available.

[12] The behavior of the strain rate is different for the Aegean sea (Figure 4a) and from the Anatolia Peninsula

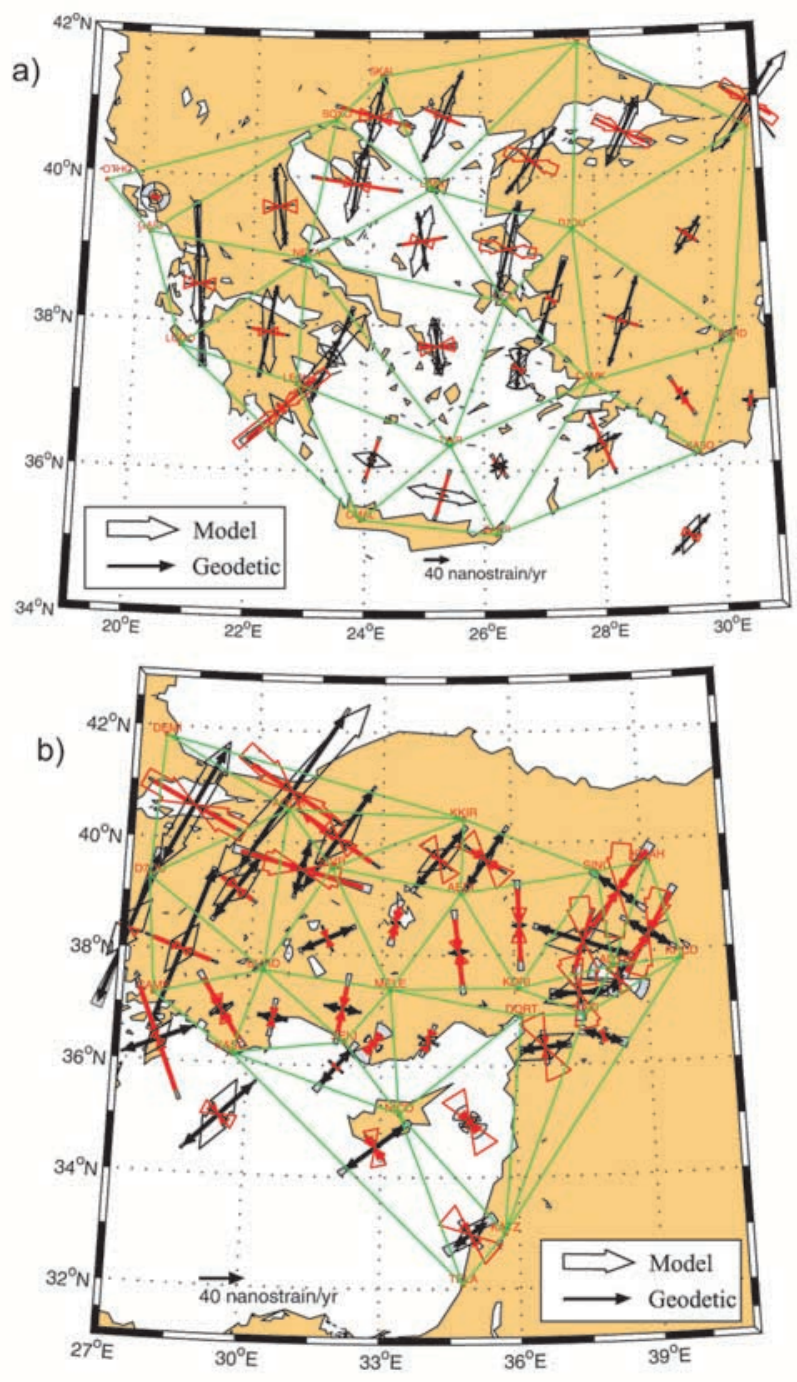

Figure 4. Geodetic (bold arrows) and modeled based on the hard rheology (empty arrows) horizontal strain rate tensor, 1 nanostrain/yr $=3.2 \times 10^{-17} \mathrm{~s}^{-1}$. Extension is represented in black and compression in red. 
(Figure 4b). In the Aegean region the close agreement between geodetic and model results, indicates that N-S oriented extension is the dominant style in the strain rate pattern, giving rise to a normal faulting tectonic regime, accordingly with the observed seismicity and stress data [Jackson and McKenzie, 1988; Rebaï et al., 1992; Mueller et al., 2000]. It is remarkable the close agreement in the eigendirections, with some deviation between the geodetic and modeled eigevalues. This N-S geodetic and modeled extension rotates in the clockwise direction to the east in Anatolia, where the largest strain rates are attained in the western part of the NAF. These large geodetic and modeled strain rates, of the order of 80 nanostrain/yr $\left(2.6 \times 10^{-15}\right.$ $\mathrm{s}^{-1}$ ), are well correlated with the high seismicity zone and high seismic strain rates of the order of $10^{-15}-10^{-14} \mathrm{~s}^{-1}$ in western Anatolia, as shown in Figure 1 between $26^{\circ}$ and $30^{\circ} \mathrm{E}$. In the same way, in the central Anatolia, it is remarkable the overlapping of the low geodetic and modeled strain rate region between $33^{\circ}$ and $36^{\circ} \mathrm{E}$ with the seismic strain rate minimum of Figure 1. To the east, the compressive component becomes higher, both in the geodetic and modeled strain rates, with deviations between these two quantities due to the proximity of the boundary where the kinematic conditions are applied, in agreement with the azimuthal misfit observed also in Figure 2.

[13] The average modeled maximum shear stress is of the order of $90-100 \mathrm{MPa}$ in western and eastern Anatolia and of $30 \mathrm{MPa}$ in central Anatolia.

[14] The gravitational potential energy, due to the crustal and lithospheric mantle variation, is self-consistently included in the modeling. However, the variation in the gravitational potential energy between western and eastern Anatolia is not high enough to induce the westward motion of Anatolia. This is induced by the key mechanisms of trench suction in the Aegean and push of Arabia.

\section{Conclusions}

[15] Comparison between geodetic and seismic deformation patterns with model predictions constrains the rheology and dynamics of Anatolia. A hard rheology, corresponding to an extreme value for the lithospheric strength [Kirby, 1983], carries into coincidence the geodetic and modeled velocity patterns in the peninsula. Geodetic and modeled strain rate patterns are in good agreement, especially in terms of the eigenvectors. The strain rate eigenvalue pattern correlates with the seismic strain rate. The model reproduces the region of high seismic strain in western Anatolia, in proximity of the $\mathrm{NAF}$, and the one of low seismic strain in the center of the peninsula. In general, the agreement between geodetic and modeled strain rates is higher than the agreement between these two strain rates and the seismic one, due to the longer time scale pertaining to the rheological model with respect to the relatively short one of the seismic data. The modeled, geodetic and seismic deformation patterns envisage the block like behavior of Anatolia.

[16] Acknowledgments. This work is supported by the COFIN2000 project of M.I.U.R "Active deformation at the northern boundary of Adria". Peter Bird is thanked for the finite element SHELLS program.
We thank Roberto Devoti, and the Telespazio and the Italian Space Agency groups, for their geodetic support and strain rate tensor calculations. We thank Ana Negredo and Anna Maria Marotta for important discussions and two anonymous referees for their constructive remarks.

\section{References}

Bird, P., Thin-plate and thin-shell finite element programs for forward dynamic modeling of plate deformation and faulting, Computers \& Geosciences, 25, 383-394, 1999.

Cianetti, S., P. Gasperini, C. Giunchi, and E. Boschi, Numerical modelling of the Aegean-Anatolian region: geodynamical constraints from observed rheological heterogeneities, Geophys. J. Int., 146, 760-780, 2001.

DeMets, C., R. G. Gordon, D. F. Argus, and S. Stein, Effect of recent revisions to the geomagnetic reversal time scale on estimates of current plate motions, Geophys. Res. Lett., 21, 2191-2194, 1994.

Devoti, R., C. Ferraro, R. Lanotte, V. Luceri, A. Nardi, R. Pacione, P. Rutigliano, C. Sciarretta, E. Gueguen, G. Bianco, and F. Vespe, Geophysical interpretation of geodetic deformations in the central Mediterranean area, in Plate Boundary Zone, Geodyn. Ser. vol. 30, edited by S. Stein and J. T. Freymueller, 57-65, AGU Washington, D. C., 2001.

Dewey, J. F., and A. M. C. Sengor, Aegean and surrounding regions: complex multiplate and continuum tectonics in a convergent zone, Geol. Soc. Am Bull., 90, 84-92, 1979.

Fernàndez, M., and G. Ranalli, The role of the rheology in extensional basin formation modelling, Tectonophysics, 282, 129-145, 1997.

Jackson, J., and D. McKenzie, The relationship between plate motions and seismic moment tensors, and the rates of active deformation din the Mediterranean and Middle East, Geophys. J. R. Astron. Soc., 93, 4573,1988 .

Jiménez-Munt, I., M. Fernandez, M. Torne, and P. Bird, The transition from linear to diffuse plate boundary in the Azores-Gibraltar region: results from a thin-sheet model, Earth. Planet. Sci. Lett., 192, 175-189, 2001.

Jiménez-Munt, I., R. Sabadini, A. Gardi, and G. Bianco, Active deformation in the Mediterranean from Gibraltar to Anatolia inferred from numerical modeling, geodetic and seismological data, J. Geophys. Res., in press, 2002.

Kirby, S. H., Rheology of the lithosphere, Rev. Geophys., 21, 1458-1487, 1983.

Kostrov, V., Seismic moment and energy of earthquakes, and seismic flow of rock, Izv. Acad. Sci. USSR Phys. Solid Earth, 1, 23-44, 1974.

Le Pichon, X., and J. Angelier, The Hellenic arc and trench system: a key to the neotectonic evolution of the eastern Mediterranean area, Tectonophysics, 60, 1-42, 1979.

Le Pichon, X., N. Chamot-Rooke, S. Lallemant, R. Noomen, and G. Veis, Geodetic determination of the kinematics of central Greece with respect to Europe: Implications for eastern Mediterranean tectonics, J. Geophys. Res, 100, 12,675-12,690, 1995.

Lundgren, P., D. Giardini, and R. M. Russo, A geodynamic framework for eastern Mediterranean kinematics, Geophys. Res. Lett., 25, 4007-4010, 1998.

McClusky, S., et al., Global Positioning System constraints on plate kinematics and dynamics in the eastern Mediterranean and Caucasus, J. Geophys. Res., 105, 5695-5719, 2000.

McKenzie, D. P., Plate tectonics of the Mediterranean region, Nature, 226, $239-243,1970$

Meijer, P. Th., and M. J. R. Wortel, Temporal variations in the stress field of the Aegean region, Geophys. Res. Lett., 23, 439-442, 1996.

Mueller, B., J. Reinecker, O. Heidbach, and K. Fuchs, The 2000 release of the World Stress Map, available online (www.world-stress-map.org), 2000.

Reilinger, R. E., et al., Global positioning system measurements of presentday crustal movements in the Arabia-Africa-Eurasia plate collision zone, J. Geophys. Res., 102, 9983-9999, 1997.

Rebaï, S., H. Philip, and A. Taboada, Modern tectonics stress field in the Mediterranean region: evidence for variation in stress directions at different scales, Geophys. J. Int., 110, 106-140, 1992.

Taymaz, T., J. Jackson, and D. McKenzie, Active tectonics of the north and central Aegean Sea, Geophys. J. Int., 106, 433-490, 1991.

Wortel, M. J. R., and W. Spakman, Subduction and slab detachment in the Mediterranean-Carpathian region, Science, 290, 1910-1917, 2000.

I. Jiménez-Munt and R. Sabadini, Geofisica, Department of Earth Sciences, University of Milan, Cicognara 7, 20129 Milano, Italy. (ivone. jimenez@unimi.it) 